\title{
Seminários Odontológicos: desenvolvendo o conhecimento, a pesquisa e o senso crítico
}

Dental Seminars: developing knowledge, research and critical thinking

Seminarios Odontológicos: desarrollo del conocimiento, investigación y sentido crítico

Flávia Akemi Nakayama Henschel ORCID: https://orcid.org/0000-0003-3186-3346

Universidade Estadual de Maringá, Brasil

E-mail: flaviaakemi95@gmail.com

Camila Fernanda Vasconcelos

ORCID: https://orcid.org/0000-0002-1597-9448

Universidade Estadual de Maringá, Brasil

E-mail: camilafv12@gmail.com

Carla Militão Ricken

ORCID: https://orcid.org/0000-0002-9174-590X

Universidade Estadual de Maringá, Brasil

E-mail: carlaricken7@gmail.com

Laura Moretto Molina

ORCID: https://orcid.org/0000-0001-9815-7775

Universidade Estadual de Maringá, Brasil

E-mail: laurammolina1@gmail.com

Letícia Caselato Ceron

ORCID: https://orcid.org/0000-0003-0818-2323

Universidade Estadual de Maringá, Brasil

E-mail: leticiacceron@gmail.com

Maicom Colombo Júnio

ORCID: https://orcid.org/0000-0001-8014-6445

Universidade Estadual de Maringá, Brasil

E-mail: maicomjr98@gmail.com

Weslley Souza Petyk

ORCID: https://orcid.org/0000-0002-4821-2200 Universidade Estadual de Maringá, Brasil

E-mail: weslleypetyk@gmail.com

Daniela Suemi Kamikawa

ORCID: https://orcid.org/0000-0002-7152-0500 Universidade Estadual de Maringá, Brasil

E-mail: danielasuemi97@gmail.com

Gabriela Steckel Neme

ORCID: https://orcid.org/0000-0003-1986-0206 Universidade Estadual de Maringá, Brasil

E-mail: gabrielasteckelneme@ gmail.com

Maria Eduarda Fernandes

ORCID: https://orcid.org/0000-0003-0867-5232 Universidade Estadual de Maringá, Brasil

E-mail: mariaeduardafernaandes@gmail.com

Mariana Podadeiro de Andrade

ORCID: https://orcid.org/0000-0001-7903-2168

Universidade Estadual de Maringá, Brasil E-mail: marianapodadeiro@gmail.com

Ana Elisa de Carvalho Manholer ORCID: https://orcid.org/0000-0002-7691-033X

Universidade Estadual de Maringá, Brasil E-mail: anaelisamanholer@gmail.com

Ana Flávia Borges Shimada

ORCID: https://orcid.org/0000-0003-1637-8772

Universidade Estadual de Maringá, Brasil

E-mail: anafshimada@gmail.com

Eduarda Letícia Pagliosa

ORCID: https://orcid.org/0000-0002-3160-7062

Universidade Estadual de Maringá, Brasil E-mail:pagliosaeduarda@gmail.com 
Leonardo Galvão da Silva Garcia

ORCID: https://orcid.org/0000-0002-5717-8040 Universidade Estadual de Maringá, Brasil E-mail: ra109875@uem.br

Luisa Gonçalves Cardoso

ORCID: https://orcid.org/0000-0002-2182-707X Universidade Estadual de Maringá, Brasil

E-mail: luisagcardoso1@gmail.com

Renata Yumi Takahashi

ORCID: https://orcid.org/0000-0003-2845-4824 Universidade Estadual de Maringá, Brasil

E-mail: reyumi.takahashi@gmail.com

Carlos Alberto Herrero de Morais

ORCID: https://orcid.org/0000-0002-5856-5608 Universidade Estadual de Maringá, Brasil E-mail: carlosherrero31@gmail.com

\begin{abstract}
Resumo
O seminário odontológico é um sistema de educação integral que permite ao aluno um conhecimento enriquecedor de diferentes assuntos, além de proporcionar formas adequadas de interdisciplinaridade. O objetivo é propiciar ao aluno melhor desenvoltura na elaboração, apresentação e oratória de seminários, promovendo a capacidade de pesquisa e a sistematização de fatos e raciocínios a respeito de assuntos da área odontológica. Além disso, o desenvolvimento de seminários permite o aprimoramento da habilidade de falar em público e redução da ansiedade, resultando em uma consequente melhora no desempenho do aluno. Nesse sentido, este artigo tem, como finalidade, relatar a experiência da atividade desenvolvida pelo Programa de Educação Tutorial (PET) do curso de Odontologia da Universidade Estadual de Maringá durante o ano de 2019, que visa à complementação da formação do petiano, denominada "Seminários Odontológicos". Os integrantes são organizados em grupos para o desenvolvimento dos seminários, relacionados à Odontologia. A atividade é aberta à toda comunidade acadêmica e a sua divulgação é realizada por meio das redes sociais. Os petianos, mediante a apresentação dos seminários, garantem uma formação interdisciplinar, interagindo com convidados e docentes, aumentando o interesse e o conhecimento por assuntos de cunho odontológico. Além disso, há o desenvolvimento da visão crítica através da experiência de banca avaliadora. É possível dizer que as ações interdisciplinares oportunizam experiências diferenciadas em um cenário inovador de ensino e aprendizagem. Esse contexto contribui para a formação dos futuros cirurgiões-dentistas, preparados para proceder atividades de forma humanizada e interligada com a capacidade de apresentação ao público.
\end{abstract}

Palavras-chave: Aprendizagem; Fala; Odontologia; Ensino.

\begin{abstract}
The dental seminar is an integral education system, which allows the student enriching knowledge of different subjects, in addition to providing adequate forms of interdisciplinarity. The objective is to provide the student with a better resource in the elaboration, presentation and oratory of seminars, promoting the ability to research and systematize the facts, reasoning and reflection around issues in the dental area. Moreover, the development of seminars allows the improvement of the ability to speak in public and reduce anxiety, with a consequent improvement in performance. Therefore, this work aims to report the experience of the activity developed by the Tutorial Education Program (PET) of the Dentistry course at the State University of Maringá, which aims to complement the training of the student, called "Dental Seminars". PET members are organized into groups for the development of seminars. The activity is open to the entire academic community and its propagation is carried out through our social networks. Petians, through the presentation of the seminars, seek to guarantee an interdisciplinary and complete training, interacting with guests and teachers, increasing interest and knowledge on various dental issues. Still, there is the development of a critical view from the experience of an evaluation board. It is possible to say that interdisciplinary actions provide differentiated experiences in an innovative teaching and learning scenario. This context contributes to the training of future dentists, prepared to carry out activities in a humanized way and interconnected with the capacity for greater skill in presenting to the public.
\end{abstract}

Keywords: Learning; Speech; Dentistry; Teaching.

\title{
Resumen
}

El seminario odontológico es un sistema educativo integral, que permite al alumno enriquecer los conocimientos, además de brindarle formas de interdisciplinariedad. El objetivo es dotar al alumno de un recurso en la elaboración, presentación y oratoria de seminarios, fomentando la capacidad de investigar y sistematizar los hechos, razonamientos y reflexiones en torno a temas del área odontológica. Además, el desarrollo de seminarios permite mejorar la capacidad de hablar en público y reducir la ansiedad, consecuentemente mejorando su rendimiento. Por ello, el objetivo del artículo es reportar la experiencia de la actividad desarrollada por el Programa de Educación Tutorial (PET) del curso de Odontología de la Universidad Estadual de Maringá durante el año de 2019, que tiene como objetivo complementar la formación del petiano, denominada "Seminarios de Odontología ". Los integrantes se 
organizan en grupos para desarrollar seminarios relacionados con la odontología. La actividad está abierta a toda la comunidad académica y su divulgación se realiza a través de redes sociales. Petianos a través de la presentación de seminarios, buscan garantizar una formación interdisciplinar y completa, interactuando con invitados y profesores, aumentando el interés y conocimiento sobre diversos temas odontológicos. También, existe el desarrollo de una mirada crítica a partir de la experiencia de una tabla de evaluación. Es posible que las acciones interdisciplinarias brinden experiencias en un escenario innovador de enseñanza y aprendizaje. Este contexto contribuye a la formación de futuros odontólogos, preparados para realizar actividades de forma humanizada e interconectados con la mayor destreza de presentación al público.

Palabras clave: Aprendizaje; Habla; Odontología; Ensenãnza.

\section{Introdução}

Ao ingressar no ensino superior, a habilidade de comunicação frente ao público e senso crítico é requerida e é de suma importância (Silva, 2003). Segundo Bolsoni et al. (2010) apresentar uma boa composição de habilidades interpessoais e uma prática bem-sucedida em oralidade em público é imprescindível para que um estudante apresente uma boa performance acadêmica e social.

Estudos apontam que as situações, no ambiente acadêmico, na qual o aluno se vê frente a uma exigência de falar em público, como apresentação de trabalhos e seminários em sala de aula ou mesmo exposição de opiniões em grupo podem produzir cenários geradores de ansiedade (Martinez et al., 2000; Oliveira \& Duarte, 2004). Em uma pesquisa realizada, a preocupação de medo de falar em público e lidar com superiores representou 90,69\% da amostra dos estudantes universitários entrevistados (Oliveira \& Duarte, 2004). Podendo, também, ser uma das principais situações de enfrentamento de casos diagnosticados como Transtorno Ansiedade Social (TAS) ou Fobia Social, que se caracteriza como o temor acentuado e persistente de conjunturas sociais ou que exijam desempenho, onde o indivíduo possui o medo de se sentir embaraçado e envergonhado (Burato et al., 2009; Hofmann \& DiBartolo, 2000).

A educação foi transmitida, de forma passiva, aos alunos, durante um longo período, sem o exercício do poder de questionar e criticar, além disso, sem gerar transformação e sem fomentar a criatividade (Freire, 1987). Atualmente, além de não reproduzir este tipo de educação, a Universidade também deve se preocupar em preparar o aluno para o mercado de trabalho e para a vida, com visão crítica e com condições de gerar novos conhecimentos (Almeida \& Costa et al., 2017).

A técnica que favorece a vivência da socialização e da construção coletiva de conhecimento é apresentada nos desenvolvimentos de seminários. A forma de planejamento consiste na formação de grupos, ao explorar assuntos e discuti-los, promovendo o intercâmbio de ideias e experiências, na realização das pesquisas e na apresentação dos trabalhos, sob a orientação de um facilitador (Rodrigues et al., 2015). Por meio de um trabalho de Bolsoni et al. (2010), os seminários se mostraram também ser uma boa ferramenta utilizada pelos professores como método de melhora do desempenho, redução da ansiedade e aprimoramento da habilidade de falar em público. Além disso, os seminários permitem a memorização das informações apresentadas pois, em função da intensa carga emocional do exercício desta técnica, o cérebro primitivo abre totalmente as barreiras que inibem a atenção (Araujo \& Santos, 2014).

No trabalho de Almeida e Costa (2017) é apontada uma preocupação a respeito das estratégias didáticas aplicadas nas universidades. O receio descrito permeia, principalmente, os métodos adotados nos seminários, que podem culminar em um trabalho fragmentado, com pouca investigação, ausência de interação e debate pelos estudantes (Almeida \& Santos, 2014; Veiga, 2006).

É essencial ressaltar que não há uma forma correta e única de desenvolver um seminário. No entanto, a estratégia principal do seminário é a organização em que ele é realizado (Gill, 2008). Nesse contexto, esse artigo tem, como objetivo, relatar a experiência da atividade desenvolvida pelo grupo PET Odontologia da Universidade Estadual de Maringá durante o ano de 2019, de cunho odontológico e que visa à complementação da formação do petiano, denominada "Seminários Odontológicos". 


\section{Metodologia}

A atividade dos seminários foi divulgada nas redes sociais do grupo PET Odontologia e ocorreu de forma aberta à comunidade acadêmica interna e externa. O evento aconteceu no Departamento de Odontologia da Universidade Estadual de Maringá. O grupo foi dividido em seis subgrupos distintos, compostos por dois ou três petianos. A partir disso, foi definido, juntamente com as datas das apresentações, qual grupo apresentaria e qual seria a banca avaliadora, dentro de um sistema de rodízio, no qual todos, em algum momento, teriam a oportunidade de fazer o papel de avaliador e, em outro, apresentador. Os petianos selecionaram temas relacionados à Odontologia e, em seguida, convidaram um docente para orientar o trabalho.

Antes de realizarem as apresentações nos dias predeterminados, cada equipe teve 25 dias, a partir do dia que foi feita a divisão dos grupos, para elaborar um resumo que foi enviado para a banca avaliadora, pré-selecionada. Após o envio do resumo, a banca tinha um período de 5 dias para aprovar a submissão ou solicitar a ressubmissão com as correções necessárias e, caso isso acontecesse, o grupo teria 5 dias para realizar as correções e o reenvio do resumo.

Segundo Pereira et al. (2018), a metodologia empregada baseou-se em uma estratégia qualitativa, utilizando-se de apresentações que ocorreram em três datas distintas ao longo do primeiro semestre. A cada dia dois grupos se apresentaram e cada apresentação teve duração de, no mínimo, 8 minutos e, no máximo, 15 minutos, com sequência de 5 minutos para dúvidas ou perguntas e 5 minutos de arguição, realizada pela banca avaliadora. A banca avaliou o assunto, resumo e a apresentação, assim como o desenvolvimento da atividade realizada pelo grupo. Essa avaliação foi feita e baseada em alguns tópicos: apresentação e desenvolvimento (AD) - 0 a 10; conhecimento do tema (CT) - 0 a 10; importância do assunto (IA) - 0 a 10; relevância científica (RC) 0 a 10; tempo (TP) - 0 ou 10; qualidade dos slides (QS) - 0 a 10; resumo - estrutura e coerência (RES) - 0 a 10. Após a banca definir todas as notas, foi determinada uma média aritmética e aplicada a nota final. Além da presença de todos os petianos, esse evento de extensão contou com a presença de docentes e discentes do Departamento de Odontologia da Universidade Estadual de Maringá e, também, de alunos de outras instituições.

\section{Resultados e Discussão}

Foram apresentados um total de seis trabalhos durante os três encontros da atividade "Seminários Odontológicos". Os petianos, mediante a apresentação dos seminários, buscaram garantir uma formação interdisciplinar e completa, interagindo com convidados e docentes, aumentando o interesse e conhecimento por diversos assuntos de cunho odontológico. A prática dos seminários é uma atividade que envolve a articulação de vários conhecimentos e aprendizagens, entre elas, o aprimoramento de práticas didáticas e o domínio de conhecimentos específicos do campo científico (Almeida \& Costa, 2017).

Os temas relacionados à Odontologia trazidos pelos grupos foram: grupo 01 - "Transplante dentário autógeno: uma opção de tratamento baseado em um relato de caso", grupo 02 - "Líquen Plano: revisão de literatura", grupo 03 - "Fotografia e videografia como recursos na Odontologia", grupo 04 - "Reabilitação estético-funcional em odontopediatria: relato de um caso clínico", grupo 05 - "Como se calcular o tempo para os retornos de manutenção periodontal? ” e o grupo 06 - "Abordagem clínica em pacientes gestantes".

O Quadro 1 indica os resultados da avaliação de cada grupo, na qual é observado que somente 2 grupos não atingiram o tempo estimado e os grupos 01 e 03 obtiveram notas mais baixas que os demais na avaliação dos seus resumos. Porém, percebemos a dedicação por parte dos petianos em estudar e conhecer, de maneira aprofundada cada tema, trazendo, assim, assuntos de relevância, com embasamento científico, para as pessoas presentes no evento. 
Quadro 1. Descrição da pontuação dos grupos.

\begin{tabular}{|c|c|c|c|c|c|c|c|c|}
\hline Grupo & AD & CT & IA & RC & TP & QS & RES & Média Final \\
\hline 01 & 10 & 10 & 9,5 & 9,5 & 10 & 10 & 8,5 & 9,64 \\
\hline 02 & 9,5 & 9,5 & 10 & 10 & 10 & 9 & 9 & 9,57 \\
\hline 03 & 9,5 & 10 & 9,5 & 9 & 0 & 9,5 & 8 & 7,92 \\
\hline 04 & 10 & 10 & 10 & 10 & 10 & 9,5 & 9,5 & 9,85 \\
\hline 05 & 10 & 9,5 & 10 & 10 & 0 & 10 & 10 & 8,5 \\
\hline 06 & 9,5 & 10 & 10 & 10 & 10 & 10 & 9,5 & 9,85 \\
\hline
\end{tabular}

Notas: AD - apresentação e desenvolvimento; CT - conhecimento do tema; IA - importância do assunto; RC - relevância crítica; TP - tempo; QS - qualidade dos slides; RES - resumo (estrutura e coerência).

Fonte: PET Odontologia.

A avaliação, juntamente com as colocações da banca avaliadora, permitiu a todos os petianos uma melhora no preparo e na apresentação dos seminários, além da importância da divulgação e da busca de temas em artigos científicos e demais fontes de conteúdo. Além disso, percebeu-se forte estímulo ao senso crítico, uma vez que cada equipe também precisou atuar como banca avaliadora. Esse maior aprofundamento é um dos objetivos presentes nos Parâmetros Curriculares Nacionais, que visam a promoção de um ensino voltado para a formação de cidadãos (Silva, 2003).

Segundo Rodrigues et al. (2015) e Rangel (2005), o seminário é uma técnica de ensino coletivo que busca a interação, o diálogo e a parceria dos envolvidos, enfatizando a troca de conhecimentos e a discussão, como meta para atingir os objetivos. Além disso, essa técnica auxilia o aluno no desenvolvimento de suas habilidades em pesquisa, autonomia, comunicação e argumentação oral (Capellato et al., 2019). Desse modo, foi possível observar que a atividade proporcionou uma maior integração e ambientação do grupo, visto que os petianos precisaram trabalhar em coletividade para elaborar e para apresentar os seminários, o que permitiu um maior enriquecimento de conhecimentos pessoais e da comunidade acadêmica com os assuntos abordados e discutidos.

Davidhizar e Cosgray (1991) considera a comunicação um instrumento básico e fundamental para aprendizagem e ensino, assim como reitera a relação com o sucesso profissional. Entretanto, falar em público é uma tarefa difícil, pois a insegurança, a ansiedade e o medo, muitas vezes, estão presentes (Lancaster, 1985). Durante a atividade, os sentimentos poderão vir como uma forma de autopreservação frente a situação, visto que o discente fica exposto a críticas e a avaliações, podendo ocasionar situações de ansiedade e estresse que podem interferir no desempenho da apresentação (Hofmann \& Dibartolo, 2000). Dessa forma, os seminários odontológicos buscam trazer essa vertente como uma forma de capacitar os participantes.

As temáticas abordadas pelos grupos e a dinâmica da atividade permitiram uma série de resultados. A curto prazo, os integrantes perceberam a necessidade de melhorar alguns pontos apontados pelos petianos avaliadores do dia, como adequações em slides, melhoras no desempenho da fala, vícios de linguagem, aprimoramento na exploração e abordagem do conteúdo e também, na maneira de se portar durante a apresentação. A médio prazo, os alunos, por meio da prática, desenvolveram habilidades na escrita, na oratória e no trabalho em equipe. Como consequência, obtiveram experiências suficientes para auxiliar os membros mais recentes e inexperientes do grupo em eventos futuros. Por fim, a longo prazo, os 
petianos adquiriram maior aptidão para apresentarem diversos trabalhos em congressos, aumentando, assim, a qualidade de seus currículos. Fato este que serviu no auxílio à conquista de vagas em residências, especializações, empregos e concursos.

\section{Considerações Finais}

Os seminários odontológicos enriquecem o conhecimento de petianos e dos demais acadêmicos, uma vez que permitem a experiência de desenvolvimento de pesquisa, a elaboração de trabalhos e o estímulo do senso crítico dos participantes. Além disso, a execução do seminário auxilia o integrante do grupo PET a lidar com a ansiedade, aprimorando sua habilidade de falar em público e, consequentemente, seu desempenho acadêmico e profissional. Destaca-se, também, a interação entre grupo PET e a graduação, aumentando o relacionamento do programa e dos seus integrantes com esse público e ao levar a uma maior visibilidade dentro da universidade.

Desta forma, mais pesquisas são necessárias para avaliar o desenvolvimento de acadêmicos em contexto de apresentações orais. Para trabalhos posteriores, sugere-se realizar uma pesquisa com grupos teste e controle sobre a melhoria na desenvoltura em sala de aula e eventos após a realização da atividade.

\section{Referências}

Almeida, I. C. L. D., \& Costa, J. R. (2017). O seminário como estratégia de ensino aprendizagem na aula universitária: redimensionando a prática pedagógica. EDUCERE, 1(8), p. 1-18.

Araujo, A. A. C., \& Santos, S. P. (2015). Olhares para o ensino em biologia: concepções de estudantes do ensino médio. In: Lima, M. S. L. et al. Didática e Prática de Ensino na relação com a Escola. Editora da Universidade Estadual do Ceará - EdUECE.

Bolsoni-Silva, A. T., Loureiro, S. R., Rosa, C. F., \& de Oliveira, M. C. F. A. (2010). Caracterização das habilidades sociais de universitários. Contextos Clínicos, 3(1), 62-75

Burato, K. R. da S., Crippa, J. A. de S., \& Loureiro, S. R. (2009). Transtorno de ansiedade social e comportamentos de evitação e de segurança: Uma revisão sistemática. Estudos de Psicologia (Natal), 14(2), 167-174. https://doi.org/10.1590/S1413-294X2009000200010

Capellato, P., Ribeiro, L. M. S., \& Sachs, D. (2019). Metodologias Ativas no Processo de Ensino-Aprendizagem Utilizando Seminários como Ferramentas Educacionais no Componente Curricular Química Geral. Research, Society and Development, 8(6), e50861090. http://dx.doi.org/10.33448/rsd-v8i6.1090

Davidhizar, R., \& Cosgray, R. (1991). Being an effective speaker. Today's OR Nurse, 13(8), 36-38.

Freire, P. (2019). Pedagogia do oprimido (75a ed.). Editora Paz e Terra.

Gil, A. C. (2008). Didática do ensino superior. Atlas.

Hofmann, S. G., \& DiBartolo, P. M. (2000). An Instrument to Assess Self-Statements During Public Speaking: Scale Development and Preliminary Psychometric Properties. Behavior therapy, 31(3), 499-515.

Lancaster, J. (1985). Public speaking can be improved. The Journal of Nursing Administration, 15(3), 31-35.

Martinez, A., Landim, A. K. P., Costa, C. R., Conilheiro, D., SA, E. S., Nunes, E. C., Santos, E. E., Batista, E. A. R., Lima, F., Santos, G. A., Santos, M. C. F., Cornachini, M,; Rego, S. C. B., \& Souza, S. F. (2000). Ansiedade social em estudantes universitários: preocupações mais emergentes. Revista Unicastelo, 3, $185-192$.

Oliveira, M. A. de, \& Duarte, Â. M. M. (2004). Controle de respostas de ansiedade em universitários em situações de exposições orais. Revista Brasileira de Terapia Comportamental e Cognitiva, 6(2), 183-200.

Pereira, A. S., Shitsuka, D. M., Parreira, F. J. \& Shitsuka, R. (2018). Metodologia da Pesquisa Científica. Editora UAB/NTE/UFSM.

Rangel, M. (2005). Métodos de ensino para a aprendizagem e dinamização das aulas (2a ed.). Papirus Editora.

Rodrigues, F. R. D. A., da Cunha, G. A. A., \& Bruno, R. C. (2015). Seminários temáticos como estratégia interdisciplinar de aprendizagem e desenvolvimento de competências em formação avançada. Enciclopédia Biosfera, 11(20), 761-771.

Silva, E. R. da. (2003). O desenvolvimento do senso crítico no exercício de identificação e escolha de argumentos. Revista Brasileira de Linguística Aplicada, 3(1), 57-68. http://dx.doi.org/10.1590/S1984-63982003000100005

Veiga, I. P. A. (2006). Técnicas de ensino: novos tempos, novas configurações. Papirus Editora. 